\title{
605 NHS-IL12 PLUS ENTINOSTAT COMBINATION EFFECTIVELY TARGETS ANTI-PD-1/PD-L1 CHECKPOINT RESISTANT MURINE TUMORS HARBORING MHC CLASS I AND ANTIGEN PROCESSING MACHINERY DEFICIENCY
}

Christine Minnar*, Paul Chariou, Kristin Hicks, Jeffrey Schlom, Sofia Gameiro. National Cancer Institute, Bethesda, MD, USA

Background Immune checkpoint blockade (ICB) has achieved unprecedented success in treating multiple cancer types. However, clinical benefit remains modest for most patients with solid malignancies with the majority having primary resistance to these therapies. Additionally, many patients that initially respond often acquire resistance. Tumor-intrinsic loss of MHC class I and aberrations in the interferon gamma (IFN $\gamma$ ) pathway have been shown to play an important role in ICB resistance. Entinostat, a class I HDAC inhibitor, has previously been shown to upregulate MHC class I molecules and antigen processing machinery (APM). NHS-IL12 (M9140), an IL12 fusion protein targeting necrotic tissue, increases anti-tumor effector functions through an influx of interferon gamma into the tumor microenvironment (TME). Here, we investigated the combination of Entinostat and NHS-IL12 in three different murine ICB-refractory tumor models (TC-1/a9, CMT.64, or RVP3) harboring varying MHC-class I and APM deficiencies.

Methods Entinostat and murine NHS-IL12 were administered to mice bearing lung TC-1/a9 (HPV16 E6/E7+), CMT.64 (lung), or RVP3 (sarcoma) tumors. Anti-tumor efficacy and survival were monitored. Comprehensive tumor and spleen immune profile analyses were carried out using flow cytometry. Tumor supernantants and sera were analyzed for cytokine and chemokine profiles. Additionally, whole transcriptomic analysis was carried out on TC-1/a9 tumors. TCGA datasets were analyzed for translational relevance.

Results We demonstrated that combination therapy elicits potent anti-tumor activity in ICB-resistant MHC-I deficient tumors through prolonged activation of cytotoxic CD8 $+\mathrm{T}$ cells. TC-1/a9 tumor-bearing athymic nude mice and depletion studies confirmed CD8 $\mathrm{T}$ cells were required for tumor growth control and survival. Importantly, we show for the first time that combination therapy synergizes to promote antigen presentation, MHC-I and APM upregulation and enrichment of IFN $\gamma$ and antigen processing pathways increasingly described as hallmarks of ICB resistance. Moreover, combination therapy promoted M1-macrophages and activated antigen presenting cells while suppressing M2-macrophages and Tregs in a tumor-dependent manner. Combination therapy induced high levels of IFN $\gamma$, IL-12, CXCL9 and CXCL13 in the TME, resulting in significant anti-tumor activity including in the IFN $\gamma$ signaling-impaired RVP3 tumor model. A biomarker signature of the mechanism involved in these studies is associated with patients' overall survival across multiple tumor types.

Conclusions Our findings provide a rationale for combining NHS-IL12 with Entinostat in the clinical setting for patients unresponsive to ICB.

Acknowledgements The authors thank Curtis Randolph for his excellent technical assistance.

http://dx.doi.org/10.1136/jitc-2021-SITC2021.605 